\title{
OS EFEITOS DOS EXERCÍCIOS FÍSICOS NA CAPACIDADE FUNCIONAL DE PACIENTES SUBMETIDAS À MASTECTOMIA: UMA REVISÃO LITERÁRIA
}

\author{
THE EFFECTS OF PHYSICAL EXERCISE IN THE FUNCTIONAL CAPACITY IN \\ PATIENTS SUBMITTED TO MASTECTOMY: A LITERARY REVIEW
}

\author{
Tiago José Nardi Gomes, ${ }^{1}$ Vívian da Pieve Antunes, ${ }^{1}$ Natália Fernandes Mezzomo, ${ }^{1}$ Letícia Castagna, ${ }^{1}$ Patrícia \\ de Moraes Costa ${ }^{2}$ \\ ${ }^{1}$ Centro Universitário Franciscano/Santa Maria-RS/Brasil. ${ }^{2}$ Universidade Federal de Santa Maria/Santa Maria-RS/Brasil \\ Autor correspondente: Letícia Castagna e-mail: leticiacastagnaa@gmail.com
}

\section{EDITORES}

Thiago Gomes Heck

(Unijuí-Brasil)

Adriane Cristina Bernat Kolankiewicz

(Unijuí-Brasil)

\section{EDITORES DE ÁREA \\ Educação \& Saúde \\ Eva Teresinha de Oliveira Boff \\ (Unijuí-Brasil)}

Fisioterapia \& Saúde

Eliane Roseli Winkelmann

(Unijuí-Brasil)

Ciências Farmacêuticas \& Saúde

Marilei Uecker Pletsch

(Unijuí-Brasil)

\section{Nutrição \& Saúde}

Lígia Beatriz Bento Franz

(Unijuí-Brasil)

Nadia Oliveira

(Unipampa-Brasil)

Ingrid Perry

(UNESC-Brasil)

Enfermagem e suas contribuições para

a prática

Adriane Cristina Kolankiewicz

(Unijuí-Brasil)

Crhis de Brum

(UFFS-Brasil)

Neila de Souza

(UFSM-Brasil)

\section{Exercício Físico \& Saúde}

Thiago Gomes Heck

(Unijuí-Brasil)

Anderson Zampier Ulbrich

(UFPR)

Editora Unijuí

Universidade Regional do Noroeste do

Estado do Rio Grande do Sul (Unijuí)

\section{RESUMO}

O câncer de mama é uma doença estigmatizada que amedronta as mulheres. A cirurgia de câncer de mama está ligada a alterações, sequelas e complicações em até $70 \%$ das mulheres acometidas, afetando sua qualidade de vida, apresentando após a cirurgia dificuldades em realizar suas atividades de vida diária. Na tentativa de minimizar os agravos físicos, psicológicos e sociais pós-tratamento do câncer existem várias práticas complementares e auxiliares ao tratamento, entre essas enfatizamos a prática da atividade física que reflete em benefícios diretos, aumentando a capacidade funcional. Este estudo visou abordar a importância do exercício físico em pacientes com câncer de mama que realizaram mastectomia e demonstrar seus benefícios. Realizou-se revisão de literatura por meio de bases de dados eletrônicas Lilacs, PEDro, Scielo, Bireme e PubMed. Vários estudos comprovam a importância do exercício para a melhora da capacidade funcional e qualidade de vida e salientam que para que apresente o efeito desejado deve ser realizado de forma regular e constante e com acompanhamento profissional. Alguns autores citam fatores que dificultam a realização dos exercícios para estas pacientes, como a falta de tempo, efeitos colaterais do tratamento, desânimo, entre outros. Essa revisão concluiu que os exercícios físicos quando realizados de maneira correta e acompanhados de um profissional conferiram efeitos positivos aos pacientes abordados, no entanto, ainda não fazem parte da rotina de muitos pacientes, justificando a necessidade de divulgação e maiores estudos sobre o tema.

Palavras-chave: Mastectomia. Fisioterapia. Exercício aeróbico. Neoplasias da mama. Atividade motora.

Submetido em: $1 \% / 3 / 2016$

Aceito em: $4 / 7 / 2016$ 


\section{Revista}

\section{Contexto}

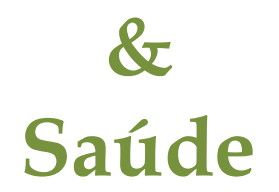

Volume 16

Número 31

2016

ISSN 2176-7114
A Revista Contexto \& Saúde é um periódico do Departamento de Ciências da Vida da Universidade Regional do Noroeste do Estado do Rio Grande do Sul (Unijuí). É um periódico semestral que tem por objetivo a divulgação da produção técnico-científica de temas relacionados à área de Ciências da Saúde.

O escopo da revista abrange a divulgação de resultados de pesquisa que contemplem avanços no processo saúde-doençacuidado e no conhecimento e aplicabilidade de novos processos químicos e biológicos em saúde.

Neste periódico, entende-se que a publicação de estudos com os aspectos epidemiológicos, assistenciais e educacionais em saúde, experimentais e aplicados é uma forma a subsidiar e qualificar a atenção à saúde de modo interdisciplinar.

\begin{abstract}
Breast cancer is a stigmatized disease that frighten women. The breast cancer surgery is linked to changes, complications and sequelae in up to $70 \%$ of affected women, affecting their quality of life, and after surgery difficulties in performing activities of daily life. In an attempt to minimize physical injuries, psychological and social after cancer treatment there are several complementary practices and aids the treatment of cancer, among these, we emphasize the physical activity that resonates in direct benefits, increasing functional capacity. This study aimed to address the importance of exercise in patients with breast cancer who underwent mastectomy and demonstrate its benefits. We conducted a literature review using electronic databases Lilacs, PEDro, SciELO, Bireme and PubMed. Several studies have confirmed the importance of exercise to improve functional capacity and quality of life and stress that to present the desired effect should be performed on a regular and continuing basis at the professional supervision. Some authors cite factors that hinder the exercises for these patients, such as lack of time, side effects of treatment, discouragement, among others. This review concluded that exercise when performed correctly and accompanied by a professional gave positive effects to patients approached, but, however, not yet part of the routine of many patients, justifying the need for disclosure and further studies on the subject .
\end{abstract}

Keywords: Mastectomy. Physical therapy specialty. Exercise. Breast neoplasms. Motor activity. 


\section{INTRODUÇÃO}

O câncer de mama é uma doença estigmatizada que amedronta as mulheres, especialmente a partir dos 40 anos. Atualmente as doenças oncológicas são consideradas problema de saúde pública pela elevada incidência, prevalência, mortalidade, gastos hospitalares e, acima de tudo, pelas consequências sobre a qualidade de vida da mulher (WHO, 2012).

A cirurgia de câncer de mama está ligada a sequelas e complicações em até $70 \%$ das mulheres submetidas a este procedimento, afetando sua qualidade de vida e resultando em dificuldades em realizar suas atividades de vida diária (AVDs) (RIBEIRO; COSTA; SANDOVAL, 2008).

Um estudo realizado em São Paulo com mulheres com câncer de mama participantes de um núcleo especializado na reabilitação física e psicossocial evidenciou que essas pacientes vivenciam alteração na capacidade funcional, o que prejudica a realização de suas atividades cotidianas e sua participação social, levando a um comprometimento na qualidade de vida (FANGEL et al., 2013).

Na tentativa de minimizar os agravos físicos, psicológicos e sociais pós-tratamento do câncer existem várias práticas complementares e auxiliares ao tratamento. Entre essas enfatizamos a prática da atividade física que reflete em benefícios diretos, aumentando a capacidade funcional e adaptações cardiorrespiratórias e musculares, por meio das respostas agudas e adaptações advindas da exigência do maior gasto energético e esforço sobre múltiplos órgãos e inclusive sobre o sistema enzimático (SAÇO; FERREIRA, 2010).

A prática de exercícios físicos relacionados com a reabilitação pós-mastectomia, bem como a orientação destes, são intervenções importantes para prevenção da limitação articular, linfedema, alterações posturais ou aderência tecidual da área cirúrgica (PRADO et al., 2004).

A capacidade funcional vai além do desempenho físico, ela retrata como o paciente conduz suas atividades diárias e reflete o quão bem ele absorve o impacto da doença em sua rotina diária; isto é individual e determinado por uma interação de variáveis clínicas e fatores psicossociais (GUERREIRO et al., 2013).

Visando ao retorno das atividades de vida diária e à melhora da qualidade de vida dessas pacientes, a prática de exercícios após intervenções cirúrgicas tem fundamental importância na recuperação da mobilidade, amplitude de movimentos e força muscular, prevenindo ou minimizando a atrofia de músculos, limitações articulares, e na tentativa de redução do surgimento de linfedema (PEDROSO; ARAÚJO; STEVANATO, 2005).

O presente estudo tem como objetivo abordar a importância do exercício físico em pacientes com câncer de mama que realizaram mastectomia, bem como demonstrar os fatores que favorecem e dificultam a prática de exercícios físicos na capacidade funcional pós-mastectomia, por meio de uma revisão literária.

\section{METODOLOGIA}

Revisão narrativa de literatura por intermédio das bases de dados Lilacs, PEDro, Scielo, Bireme e PubMed. As buscas por artigos científicos inicialmente foram realizadas com os seguintes descritores: Mastectomia, Fisioterapia, Exercício aeróbico, Neoplasias da mama e Atividade motora, com seus respectivos termos em inglês: Mastectomy, Physical Therapy Specialty, Exercise, Breast Neoplasms, Motor activity. Após foi realizada uma combinação dos descritores, enfatizando o exercício físico em pacientes mastectomizadas. Foram utilizados artigos em inglês e português. A revisão abrangeu as publicações de 2004 a 2014, este último ano até 
o mês de outubro). Foram incluídos artigos completos, disponíveis on-line, na íntegra, que se encaixassem no tema da pesquisa. Foram excluídos estudos de pesquisa básica, em animais e idosas.

\section{RESULTADOS}

A pesquisa realizada nas bases de dados eletrônicas identificou 110 referências, das quais grande parte não correspondeu ao tema central da pesquisa, sendo excluídas, resultando, portanto, em 21 trabalhos no período de 2004 a 2014. Os principais fatores que favorecem ou dificultam a prática de exercícios físicos estão descritos no Quadro 1.

QUADRO 1 - Descrição dos autores e ano de publicado; desenhos dos estudos; desfecho final após o treinamento e dificuldades enfrentadas para realizar o treinamento das pacientes

\begin{tabular}{|c|c|c|c|}
\hline Autor, Ano & $\begin{array}{l}\text { Desenho do } \\
\text { estudo }\end{array}$ & Desfecho com o treinamento & $\begin{array}{c}\text { Dificuldades para realizar o } \\
\text { treinamento }\end{array}$ \\
\hline Prado et al., 2004 & $\begin{array}{l}\text { Ensaio Clínico } \\
\text { Randomizado }\end{array}$ & $\begin{array}{l}\text { Melhora da condição física, fortalecimento } \\
\text { muscular, do movimento dos braços, } \\
\text { sensação de prazer e disposição, } \\
\text { conhecimento sobre a importância da prática } \\
\text { dos exercícios, acompanhamento } \\
\text { profissional, suporte de familiares. }\end{array}$ & $\begin{array}{l}\text { Falta de força de vontade e } \\
\text { cansaço. }\end{array}$ \\
\hline Daley et al., 2007 & $\begin{array}{l}\text { Ensaio Clínico } \\
\text { Randomizado }\end{array}$ & $\begin{array}{l}\text { Horário livre para a realização dos } \\
\text { exercícios, acompanhamento profissional, } \\
\text { envolvimento familiar. }\end{array}$ & $\begin{array}{c}\text { Afazeres } \\
\text { domésticos/ocupações } \\
\text { familiares, compromisso de } \\
\text { trabalho, duração e tipo de } \\
\text { tratamento. }\end{array}$ \\
\hline Ott et al., 2004 & $\begin{array}{l}\text { Ensaio Clínico } \\
\text { Randomizado }\end{array}$ & $\begin{array}{l}\text { Informação e percepção dos benefícios, } \\
\text { acompanhamento profissional, compromisso } \\
\text { na realização dos exercícios. }\end{array}$ & $\begin{array}{l}\text { Não relaciona as barreiras } \\
\text { encontradas à pratica de } \\
\text { exercícios. }\end{array}$ \\
\hline Rogers et al., 2006 & $\begin{array}{l}\text { Ensaio Clínico } \\
\text { Randomizado }\end{array}$ & $\begin{array}{c}\text { Percepção dos benefícios do exercício sobre } \\
\text { o sistema cardiovascular, aumento de força } \\
\text { muscular, perda de peso. }\end{array}$ & $\begin{array}{l}\text { Falta de disciplina, falta de } \\
\text { tempo, falta de interesse, } \\
\text { efeitos colaterais da } \\
\text { quimioterapia, desânimo e } \\
\text { afazeres domésticos. }\end{array}$ \\
\hline $\begin{array}{l}\text { Gutiérrez et al., } \\
2007\end{array}$ & $\begin{array}{l}\text { Ensaio Clínico } \\
\text { Randomizado }\end{array}$ & $\begin{array}{l}\text { Presença de profissionais especializados, } \\
\text { grupos de apoio, suporte familiar, reforço das } \\
\text { informações em materiais ilustrativos. }\end{array}$ & $\begin{array}{l}\text { Dor, ardência, medo de afetar } \\
\text { a cirurgia, repuxo, sensação } \\
\text { de peso, fraqueza, mal-estar, } \\
\text { falta de sensibilidade na } \\
\text { região da cirurgia e mãos, } \\
\text { falta de vontade. }\end{array}$ \\
\hline $\begin{array}{l}\text { Courneya et al., } \\
2008\end{array}$ & $\begin{array}{l}\text { Ensaio Clínico } \\
\text { Randomizado }\end{array}$ & $\begin{array}{l}\text { Supervisão individual, flexibilidade de } \\
\text { horário e local dos exercícios, experiência } \\
\text { anterior com exercícios, menor depressão. }\end{array}$ & $\begin{array}{l}\text { Efeitos colaterais do } \\
\text { tratamento. }\end{array}$ \\
\hline
\end{tabular}

FONTE: Organizado pelos autores 


\section{DISCUSSÃO}

De acordo com estudo realizado pelo serviço de fisioterapia do Inca, entre as condutas fisioterapêuticas no tratamento das complicações radioterápicas e quimioterápicas estão os exercícios moderados de baixo impacto (caminhadas de 20 a 30 minutos), que auxiliam na melhora da fadiga (BERGMANN et al., 2006).

Após a cirurgia, as pacientes passam a ter uma nova realidade do esquema corporal, pois ocorrem alterações importantes em âmbito anatômico, fisiológico e funcional que podem vir acompanhadas de dores, mudança no esquema corporal, prejuízos na amplitude de movimento do ombro, resultando em um impacto negativo nas atividades do cotidiano e alterando sua maneira de sentir e vivenciar o corpo. Com base nestas alterações, a reabilitação física por meio da Fisioterapia torna-se primordial (RIBEIRO et al., 2006; DANTAS et al., 2013).

A prática de exercícios físicos associados à recuperação pós-mastectomia é descrita como benéfica, pois tem um efeito psicológico positivo no humor, a melhora da capacidade funcional e qualidade de vida, estimulando também o apetite dessas mulheres (OLIVEIRA; CHRISTOS, 1997).

No trabalho realizado por Prado et al. com 30 mulheres, observou-se que os benefícios percebidos sobre a prática do exercício físico foram a melhora na disposição, ânimo, energia e bem-estar, benefício nas articulações, melhora dos movimentos dos braços e ombros, fortalecimento dos músculos, prevenção do linfedema, ajuda na saúde mental, melhora na qualidade do sono e sensação de prazer, porém houve percepção de dificuldades como a falta de força de vontade, cansaço, falta de tempo e recursos financeiros. Os achados deste estudo reforçam a necessidade de os profissionais de saúde buscarem estratégias que motivem a prática da atividade física (PRADO et al., 2004).

Outros autores também citam fatores que dificultam a realização dos exercícios, como a falta de interesse, condições de saúde, os efeitos colaterais do tratamento, principalmente a quimioterapia, os compromissos de trabalho e o desânimo; outros fatores que dificultam são a dor e o desconforto, o receio de afetar a cirurgia, a sensação de peso, fraqueza e mal-estar e a falta de sensibilidade na região da cirurgia e nas mãos (SANT'ANNA et al., 2010; GUTIÉRREZ et al., 2007).

Alguns benefícios dos exercícios são elencados por Moraes et al. em publicação de 2014, como a elevada concentração dos hormônios corticosteroideos e de citocinas induzidas pelo exercício físico, aumentando a resposta imunológica, normalmente deprimida durante o desenvolvimento neoplásico (MORAES; CURLE; ILDEFONSO, 2014).

Outro achado de Moraes, Curle e Ildefonso foi que o exercício físico executado no tempo de 30 a 60 minutos numa intensidade moderada, reduziu $20 \%$ a $30 \%$ o risco de desenvolvimento de outro tipo de câncer, como cólon, quando comparadas às mulheres inativas (MORAES; CURLE; ILDEFONSO, 2014).

Outros benefícios do exercício físico foram apontados por Repka et al. em estudo de 2014, com 170 pacientes com câncer de mama que após três meses de exercício físico realizado três vezes por semana fez com que a frequência cardíaca, os índices de fadiga e a pressão arterial sistólica e diastólica fossem reduzidas significativamente.

Para que os exercícios apresentem os efeitos desejados é necessário que sejam realizados de forma regular e constante, e esses benefícios do exercício somente podem ser experimentados quando ocorre a participação 
regular, com acompanhamento profissional, grupos de apoio e suporte familiar (PRADO et al., 2004; GUTIÉRREZ et al., 2007).

A realização dos exercícios permitirá uma melhor condição funcional do membro superior e do nível de condicionamento, tanto pela manutenção quanto pelo incremento da amplitude de movimento e força muscular. Além disso, o exercício contribui para o aumento da autoestima e percepção da capacidade funcional das mulheres que se sentem aptas à realização das atividades por elas desenvolvidas antes da cirurgia (PRADO et al., 2004; GUTIÉRREZ et al., 2007; ROGERS et al., 2006).

Um estudo de Czerniak, Demuth e Skrzypczak (2014), com o objetivo de determinar a relação entre atividade física e composição corporal em mulheres saudáveis e mastectomizadas relatou que as mulheres mastectomizadas apresentaram índice de massa corporal e nível de massa gorda maiores que as mulheres saudáveis e após a realização de atividades físicas conseguiram atingir o mesmo nível de massa de gordura que as mulheres saudáveis.

Moraes et al. corroboram com estes achados e ainda mostram que o exercício físico aumenta o consumo de oxigênio, marcador da capacidade funcional, em $40 \%$, reduzindo a náusea, a depressão e a fadiga, consequentemente melhorando a qualidade de vida em mulheres com câncer de mama (MORAES; CURLE; ILDEFONSO, 2014).

O crescimento tumoral promove alterações no metabolismo dos indivíduos com câncer, como diminuição do peso corpóreo, redução muscular, aumento da fadiga, aumento da resistência da insulina, falta de ânimo, indisposição e mal-estar. O exercício físico quando bem orientado e supervisionado pode contribuir para uma melhora na qualidade e expectativa de vida, melhora na resposta do sistema imune e dos mecanismos antioxidantes, aumento da capacidade cardiorrespiratória, benefícios nas articulações, fortalecimento dos músculos. Dessa maneira, a ideia de repouso absoluto para pacientes pós-mastectomia só traz declínio da capacidade funcional, devendo ser evitado o mais possível (MORAES; CURLE; ILDEFONSO, 2014; DIETTRICH et al., 2006).

\section{CONSIDERAÇÕES FINAIS}

Por meio dessa revisão de literatura encontramos observações positivas referentes aos exercícios físicos, tais como a importância para prevenção da limitação articular, linfedema, alterações posturais, aderência tecidual da área cirúrgica, melhora na disposição, ânimo, energia e bem-estar, benefício nas articulações, melhora dos movimentos dos braços e ombros, fortalecimento dos músculos, auxiliando assim na melhoria da capacidade funcional, qualidade de vida e logo, na reabilitação pós-mastectomia. Tal prática, no entanto, ainda não faz parte da rotina de muitos pacientes, necessitando avançar o conhecimento de como a prática de exercícios físicos pode ajudar na reabilitação das mastectomizadas, assim como difundir mediante pesquisas o papel do fisioterapeuta neste contexto.

\section{REFERÊNCIAS}

BERGMANN, A. et al. Physical Therapy in Breast Cancer: clinical protocol at the Cancer Hospital III/Inca. Revista Brasileira de Cancerologia, v. 52, n. 1, p. 97-109, 2006.

COURNEYA, K. S. et al. Predictors of supervised exercise adherence during breast cancer chemotherapy. Medicine \& Science in Sports \& Exercise, v. 40, n. 6, p. 1.180-1.187, 2008.

CZERNIAK, U.; DEMUTH, A.; SKRZYPCZAK, M. Associations of physical activity and inactivity with body tissue composition among healthy Polish women and women after mastectomy. Journal of Comparative Human Biology, v. 65, n. 5, p. 423-431, 2014. 
DALEY, A. J. et al. Determinants of adherence to exercise in women treated of breast cancer. European Journal of Oncology Nursing, v. 11, n. 5, p. 392-399, 2007.

DANTAS, S. M. et al. Quality of Life and Shoulder Motion after Surgery for Breast Cancer: Physical Therapy Focus. Revista Brasileira de Cancerologia, v. 59, n. 3, p. 419-426, 2013.

DIETTRICH, S. H. C. et al. Efeitos de um programa de caminhada sobre níveis de fadiga em pacientes com câncer de mama. Revista Brasileira de Ciências e Movimento, v. 14, n. 1, p. 7-12, 2006.

FANGEL, L. M. V. et al. Qualidade de vida e desempenho de atividades cotidianas após tratamento das neoplasias mamárias. Acta Paulista de Enfermagem, v. 26, n. 1, p. 93-100, 2013.

GUERREIRO, V. et al. Capacidade funcional de pacientes submetidas à reconstrução mamária com retalho musculocutâneo de latíssimo do dorso. Revista Brasileira de Cirurgia, v. 28, n. 3, p. 367-274, 2013.

GUTIÉRREZ, M. G. R. et al. Adesão de mulheres mastectomizadas ao início precoce de um programa de reabilitação. Acta Paulista de Enfermagem, v. 20, n. 3, p. 249-254, 2007

MORAES, C. A.; CURLE, B. C. S.; ILDEFONSO, L. O. Benefícios do exercício físico em portadores de câncer. Revista de Saúde e Biologia, v. 9, n. 2, p. 83-92, 2014.

OLIVEIRA, S. A.; CHRISTOS, P. J. The epidemiology of physical activity and cancer. Annals of the New York Academy of Sciences, v. 833, p. 79-90, 1997.

OTT, C. D. et al. Facilitative strategies psychological factors and strength/ weight training behaviors in breast cancer survivors who are at risk for osteoporosis. Orthopaedic Nursing Journal. v. 23, n. 1, p. 45-55, 2004.

PEDROSO, W.; ARAÚJO, M. B.; STEVANATO, E. Atividade física na prevenção e na reabilitação do câncer. Revista de Educação Física, v. 11, n. 3, p. 155-160, 2005.

PRADO, M. A. S. et al. A prática da atividade física em mulheres submetidas à cirurgia por câncer de mama: percepção de barreiras e benefícios. Revista Latino-Americana de Enfermagem, v. 12, n. 3, p. 494-502, 2004.

REPKA, C. P. et al. Cancer Type Does Not Affect Exercise-Mediated Improvements in Cardiorespiratory Function and Fatigue. Integrative Cancer Therapies, v. 13, n. 6, p. 473-481, 2014.

RIBEIRO, M. C. S. A. et al. Perfil sociodemográfico e padrão de utilização de serviços de saúde para usuários e não-usuários do SUS - PNAD 2003. Ciência \& Saúde Coletiva, v. 11, n. 4, p. 1.011-1.022, 2006.

RIBEIRO, R. L.; COSTA, R. L.; SANDOVAL, R. A. Conduta Fisioterápica no Linfedema Pós-Mastectomia por Câncer de Mama. Revista Eletrônica da Faculdade de Montes Belos, v. 3, n. 1, p. 1-22, 2008.

ROGERS, L. Q. et al. Exercise barrier and task self-efficacy in breast cancer patients during treatment. Supportive Care in Cancer, v. 14, n. 1, p. 84-90, 2006.

SAÇO, L. F.; FERREIRA, E. L. Mulheres com câncer e sua relação com a atividade física. Revista Brasileira de Ciências e Movimento, v. 18, n. 4, p. 11-17, 2010.

SANT'ANNA, D. K. et al. Adesão à prática de exercícios para reabilitação funcional de mulheres com câncer de mama: revisão de literatura. Ciencia y enfermeira, v. 16, n. 1, p. 97-104, 2010.

WORLD HEALTH ORGANIZATION. WHO. Cancer. Note descriptive n. 297. Disponível em: <http://www.who.int/mediacentre/ factsheets/fs297/es/index.html3>. Acesso em: 20 fev. 2012. 